\title{
INTELLIGENT MODIFIED DIFFERENCE EXPANSION FOR REVERSIBLE WATERMARKING
}

\author{
Hirak Kumar Maity ${ }^{1}$ and Santi Prasad Maity ${ }^{2}$ \\ ${ }^{1}$ Department of Electronics and Communication Engineering, College of Engineering and \\ Management, Kolaghat, West Bengal, India \\ hirakmaityegmail.com \\ ${ }^{2}$ Department of Information Technology, Bengal Engineering and Science University, \\ Shibpur, West Bengal, India \\ santipmaitydit.becs.ac.in
}

\begin{abstract}
A Reversible Watermarking $(R W)$ using Difference Expansion $(D E)$ algorithm plays an important role on content authentication for highly secured medical and military images. According to this algorithm, the least significant bit (LSB) of inter-pixel differences (between a pair of neighboring pixel) is used to embed secret data. It is seen that none of the DE works focuses on structural information retentions for the watermarked image at high embedding capacity. Moreover, security measure of the hidden data is not investigated under distortion constraint scenario. To this aim, a modification in DE is proposed that not only increase embedding space but also makes little change in structure and contrast comparison under similar luminance background. In our approach an image is partitioned into edge, smooth and texture region using computed gradient magnitude obtained through sobel's operator followed by adaptive distortion control. Simulation results shown that, this simple yet effective modification leads to better security of the hidden data.
\end{abstract}

\section{KEYWORDS}

Reversible Watermarking, Difference Expansion, PSNR, SSIM, KLD and Distortion Control.

\section{INTRODUCTION}

Broadly we can classify reversible or lossless watermarking (RW) into three categories: 1) fragile watermarking 2) semi fragile watermarking and 3) robust watermarking [1]. From the literature review we have seen that most of the RW scheme belongs to fragile watermarking technique. Fragile watermarking is a type of watermarking technique which embeds secret information called watermark into an image that is not readable anymore if the content gets altered by any kind of intentional on unintentional attacks. And the term 'semi' indicates if it can sustain some attacks, moreover if any watermark can sustained with all kind of attacks is called robust watermarking technique. Fragile watermarking is the subject of many applications like content authentication. RW is a special case of digital watermarking with an intriguing feature that when the watermarked content has been authenticated, one can remove the watermark to retrieve the exactly same original information. Such reversibility is highly desirable in case of high secured data communication especially for medical and military applications where even small amount of distortion is not permissible.

Performance improvement of RW methods for digital images has three major requirements: a high embedding payload, low complexity and a good visual quality. It this paper we mostly look at a high payload scheme with a small complexity. Literature on reversible watermarking is quite

DOI : 10.5121/ijma.2012.4408 
The International Journal of Multimedia \& Its Applications (IJMA) Vol.4, No.4, August 2012

rich by this time [1], [3]. Among them, Tian's difference expansion (DE) scheme [2], [4] is quite popular one, where redundancy present in the image content is used to embed reversibly the payload. It is seen that none of the DE works focuses on structural information retentions for the watermarked image at high embedding capacity. Majority of them had tried to explore the redundancy in digital images to achieve very high embedding capacity with low distortion. Moreover, there is no provision on distortion control analysis in DE algorithms [2], [4]. This paper makes simple but effective modification of DE that improves data hiding capacity with much better visual and statistical invisibility of the hidden data. We classify an image into smooth, edge and texture region using the component gradient magnitude [5]. Depending on the amount occupied by each region we have found out their corresponding weight factor. On the basis of that we have found out the three different error control threshold for individual regions. Accordingly distortion control is set in different values in different regions. Furthermore, in the literature of DE; security measure of the hidden data is not investigated under distortion constraint scenario. To this aim, a simple yet effective modification in DE is suggested that not only increases embedding space but also makes little change in structure and contrast comparison under similar luminance perspective. Modification also makes a difference in relative entropy between the host and watermarked data that in turn leads to higher security for the hidden data.

The rest of the paper is organized as follows: Section 2 presents a brief literature review on RW. Section 3 introduces several mathematical models used in this work for watermarking performance assessment. Section 4 presents gradient based image partitioning for distortion control. Section 5 discussed with proposed algorithm and section 6 presents performance evaluation with discussion. Finally conclusions are drawn in Section 7.

\section{LITERATURE REVIEW}

Jun Tian [2], [4] first proposed difference expansion (DE) algorithm. According to Tian's scheme, the least significant bit (LSB) of inter-pixel differences (between a pair of neighbouring pixel) is used to embed data. In principle, the redundancy present in digital images is used to achieve a high-capacity and low-distortion reversible watermarking. Later on, Alattar et al. [6] extended Tian's scheme by using DE of spatial and cross spectral triplets instead of pixel pairs which increase the hiding ability. Tian's algorithm allows embedding of one bit in every pair of pixels, whereas Alatter's algorithm [6] embeds two bits in every triplet. Soon after Alattar et al. [7] extended Tian's scheme in different way by using DE transform of quads and able to embed two bits in every quads. The amount of data that can be embedded into the host image depends largely on the characteristics of image. So this technique is not commonly acceptable. Subsequently, Alattar et al. [8] proposed another method based on generalized DE method with integer transform where more differences were available for expansion and require low cost to record overhead information. Wang et al. [9] developed a novel scheme based on vector map which reduces the size of the location map. Lin et al [10] proposed location map free reversible data hiding technique based on DE algorithms. Later, Hu et al. [11] presented another DE scheme which demands efficient payload dependent overflow location map which has good compressibility than earlier algorithms. It contains two types of overflow location map: one from embedding and another from shifting.

\section{Mathematical Models for Watermark ASSESSMENT}

This section briefly introduces different quantitative measures used in this work for quality assessment of watermarking methods.

\subsection{PSNR and MSE}

The simplest, oldest and most widely used technique to quantify image/video signal quality is the mean squared error (MSE). Mathematically it is defined as: 
The International Journal of Multimedia \& Its Applications (IJMA) Vol.4, No.4, August 2012

$$
M S E=\frac{1}{M N} \sum_{i=0}^{M-1} \sum_{j=0}^{N-1}(u-v)^{2}
$$

Where, two images $\mathrm{u}$ and $\mathrm{v}$ having size $\mathrm{M} \times \mathrm{N}$, one of them is the noisy (watermarked) approximation of the other (original) one. Peak-signal-to noise-ratio (PSNR) used measure to quantify visual distortion made by watermarking process as well as different attack operations. Mathematically for an 8 bit gray scale image it is defined as:

$$
P S N R=10 \log _{10}\left(\frac{255^{2}}{M S E}\right)
$$

\subsection{SSIM and MSSIM}

Structural similarity index metric (SSIM) is based on the structural information of the image and provides a good measure for different kinds of images, from natural scenes to medical images [12]. It compares local patterns of pixel intensities that have been normalized for luminance and contrast. SSIM index between two images $u$ and $v$ have three levels of comparisons and is defined as follows:

$$
\operatorname{SSIM}(u, v)=[l(u, v)]^{\alpha} \cdot[c(u, v)]^{\beta} \cdot[s(u, v)]^{\gamma}
$$

where, $1, \mathrm{c}$ and $\mathrm{s}$ are the luminance, contrast, structural comparisons between two images $\mathrm{u}$ and $\mathrm{v}$, and are given by the expressions as follows:

$$
l(u, v)=\frac{2 \mu_{u} \mu_{v}+C_{1}}{\mu_{u}^{2}+\mu_{v}^{2}+C_{1}}, c(u, v)=\frac{2 \sigma_{u} \sigma_{v}+C_{2}}{\sigma_{u}^{2}+\sigma_{v}^{2}+C_{2}} \text { and } s(u, v)=\frac{\sigma_{u v}+C_{3}}{\sigma_{u} \sigma_{v}+C_{3}}
$$

Where, $\mathrm{C}_{1}, \mathrm{C}_{2}$ and $\mathrm{C}_{3}$ are three constants. The symbols $\mu$ and $\sigma$ are the local mean intensity and local standard deviation of an image. The symbol $\sigma_{\mathrm{uv}}$ is the local covariance coefficient between the images $u$ and $v$. If $\alpha=\beta=\gamma=1$ and $c_{3}=c_{2} / 2$ are considered, it can be rewritten as:

$$
\operatorname{SSIM}(u, v)=\frac{\left(2 \mu_{u} \mu_{v}+C_{1}\right)\left(2 \sigma_{u v}+C_{2}\right)}{\left(\mu_{u}^{2}+\mu_{v}^{2}+C_{1}\right)\left(\sigma_{u}^{2}+\sigma_{v}^{2}+C_{2}\right)}
$$

The overall value is obtained from the mean of the local SSIM, called MSSIM.

\subsection{KLD}

Kullback-Leibler distance (KLD) is one important security measure between two probability distribution function $\mathrm{p}_{0}$ and $\mathrm{p}_{1}$ those are closely related [13]. It used widely in steganography for security measure of the hidden data. It is a natural distance function from a "true" probability distribution, $\mathrm{p}_{1}$, to a "target" probability distribution, $\mathrm{p}_{0}$. It is frequently used as a measure of 'distance' from information theory viewpoint. If $\mathrm{p}_{0}$ and $\mathrm{p}_{1}$ are two probability densities, then KLD is defined as for continuous functions:

$$
K L D\left(p_{1} \| p_{0}\right)=\int p_{1}(x) \log \frac{p_{1}(x)}{p_{0}(x)} d x
$$

The log function has base 2. For discrete (not necessarily finite) probability distributions, the KLD is defined to be:

$$
K L D\left(p_{1}, p_{0}\right)=\sum_{i} p_{1 i} \log _{2}\left(\frac{p_{1 i}}{p_{0 i}}\right)
$$


The International Journal of Multimedia \& Its Applications (IJMA) Vol.4, No.4, August 2012

Where, $p_{1}=\left\{p_{11}, p_{12}, \ldots ., p_{1 n}\right\}$ and $p_{10}=\left\{p_{01}, p_{02}, \ldots ., p_{0 n}\right\}$. As mentioned earlier, this is used here to quantify the security i.e. statistical invisibility of the hidden data.

\section{Image Partitioning AND Distortion CONTROL}

It is seen that a natural images posses different characteristics like smooth, edge, texture region etc. and different regions are in a different way sensitive to embedding distortion. This needs adaptive watermark power control so that overall structure of the watermarked image is less affected. In automatic identification of image regions, it is difficult to find threshold gray values due to imprecise and uncertainties in gray values. Here, we partition an image into three components using the computed gradient magnitudes [5]; gives a simple image partition method that we used to obtain different image regions. The details are given in the next subsections.

\subsection{Image partitioning}

We assume here that an image contains edge, smooth and texture regions. The image is portioned into three different regions according to the following rules:

1) The gradient map of an image is generated by using Sobel's operator, that has two mask of

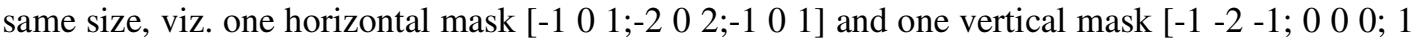
2 1], for each pixel (P) with location (i,j), its gradient vector is defined as $\bar{M}=\left\{d x_{i, j} . d y_{i, j}\right\}$. Where $d x_{i, j}$ and $d y_{i, j}$ are obtained by vertical edge mask and horizontal edge mask, respectively.

2) Next find out the maximum gradient magnitude value $g_{\max }$ computed over the original image.

3) Consider two different threshold value $T H_{1}=0.12 g_{\max }$ and $T H_{2}=0.06 g_{\max }$.

4) Assign pixels as belonging to edge, texture and smooth regions as follows:

Consider the gradient at coordinate $(i, j)$ on the original image is given by $g(i, j)$.

- If $g(i, j)>\mathrm{TH}_{1}$, then the pixel is consider as an edge pixels.

- If $g(i, j)<\mathrm{TH}_{2}$, then the pixel is regarded as being part of a smooth region.

- Otherwise, the pixel is regarded as being in a texture region.

\subsection{Error distortion control}

The pixel intensity difference value between the host and the watermarked image is called error which causes visual distortion. To control or minimize this distortion we use a predefined error threshold $(\Delta)$. If $(x, y)$ is the original pixel intensity value get modified to $\left(x^{\prime}, y^{\prime}\right)$ after watermark embedding, the error is then $\left|x-x^{\prime}\right|$ on $\mathrm{x}$ and on $\mathrm{y}$ is $\left|y-y^{\prime}\right|$. For low data hiding bit-rate, distortion control is necessary in order to reduce the distortions introduced by the watermarking. According to this mechanism, any pixel pair will be transformed if it satisfies the following conditions:

$$
\left|x-x^{\prime}\right|<\Delta \text { and }\left|y-y^{\prime}\right|<\Delta
$$

Using above subsection, we partition image into smooth, edge, texture regions and can set

distortion as $\Delta_{1}, \Delta_{2}$ and $\Delta_{3}$, respectively such that $\Delta=\frac{\Delta_{1}+\Delta_{2}+\Delta_{3}}{3}$. To find out the $\Delta$ value of different regions i.e. $\Delta_{1}, \Delta_{2}$ and $\Delta_{3}$, we have considered weight factor of each regions, which comes with their corresponding amount occupied by each region with respect to the whole region of the original image. It is assumed that overall embedding distortion using a global mean $\Delta$ value would remain similar for $\Delta_{1}, \Delta_{2}$ and $\Delta_{3}$ chosen adaptively in respective regions. 
The International Journal of Multimedia \& Its Applications (IJMA) Vol.4, No.4, August 2012

\section{Proposed Modified DE Algorithm}

This section presents the proposed watermarking scheme. For an 8 bit gray scale image, let $\mathrm{x}, \mathrm{y}$ is a pixel pair bounded by its intensity value within the range of 0 and 255. Then the forward integer transform is given by:

$$
x^{\prime}=\left\lfloor\frac{x+y}{2}\right\rfloor, y^{\prime}=\left\lfloor\frac{x-y}{2}\right\rfloor
$$

Where $x^{\prime}$ and $y$ are the forward transform pair and the symbol [.] indicates floor function meaning "the greatest integer less than or equal to". The inverse integer transform of (9) is given by:

$$
x^{\prime \prime}=x^{\prime}+y^{\prime}, y^{\prime \prime}=x^{\prime}-y^{\prime}
$$

According to the general methodology of DE, we can embed a watermark bits (w) to the LSB position of $y^{\prime}$ using the following rule:

$$
y_{1}^{\prime}=2 y^{\prime}+w
$$

Where w can be 0 or 1 depending on the watermark bits. The watermarked pixel pairs will be represented by:

$$
x_{1}^{\prime \prime}=x^{\prime}+y_{1}^{\prime}, y_{1}^{\prime \prime}=x^{\prime}-y_{1}^{\prime}
$$

Using these transformations the original image is transferred to watermarked image. The next half of the work is related with watermark extraction to get back original image from watermarked image. At this point watermarked image is the input, by applying (9) to the marked pixel pairs, following pixel pair would be obtained.

$$
x_{2}^{\prime}=\left\lfloor\frac{x_{1}^{\prime \prime}+y_{1}^{\prime \prime}}{2}\right\rfloor, y_{2}^{\prime}=\left\lfloor\frac{x_{1}^{\prime \prime}-y_{1}^{\prime \prime}}{2}\right\rfloor
$$

Now watermark bit (b) is retrieved by discarding the LSB of $y_{2}^{\prime}$ and the rest of the part $y_{2}^{\prime \prime}$ will be considered for further operation.

$$
y_{2}^{\prime \prime}=\left\lfloor\frac{y_{2}^{\prime}}{2}\right\rfloor
$$

Retrieved watermark bits (b) will be same with embedded watermark (w) due to its reversibility. Now the original pair of pixel would be back by simply combing (12), (13) and (14).

$$
x_{2}=x_{2}^{\prime}+y_{2}^{\prime \prime}, y_{2}=x_{2}^{\prime}-y_{2}^{\prime \prime}
$$

According to the condition of reversibility the intensity value of the retrieved pixel should be same with its corresponding original one, i.e. $x_{2}=x$ and $y_{2}=y$. The whole retrieving/ reconstruction process will be blind in nature i.e. without the use of the original image. In order to confined its applicability in 8 bit gray scale image, $x$ and $y^{\prime}$ should satisfy the conditions: $0 \leq\left(x^{\prime \prime}, y^{\prime \prime}\right) \leq 255$ to avoid overflow or underflow problem. Mathematically,

$$
0 \leq\left(x^{\prime}+y^{\prime}\right) \leq 255 \text { and } 0 \leq\left(x^{\prime}-y^{\prime}\right) \leq 255
$$

The above conditions can be expressed as: 
The International Journal of Multimedia \& Its Applications (IJMA) Vol.4, No.4, August 2012

$$
y^{\prime} \leq \min \left(\left(255-x^{\prime}\right), x^{\prime}\right)
$$

\subsection{Condition of changeability and expandability}

According to DE theorem, newly embedded binary digits (b) will be placed into LSB position of the difference value $y^{\prime}$, i.e. expandable difference value after placing the embedded bit will be: $\ddot{y}=2 y^{\prime}+b$. To avoid overflow or underflow problem, it should satisfy the following conditions:

$$
\ddot{y} \leq \min \left(\left(255-x^{\prime}\right), x^{\prime}\right) \text { or } 2 y^{\prime}+b \leq \min \left(\left(255-x^{\prime}\right), x^{\prime}\right)
$$

Where, $\mathrm{b}$ can be 0 or 1 and the above condition is called 'condition of expandability'.

Similarly, without making overflow or underflow, any difference value will be changeable if and only if it satisfies condition (17) after embedding, i.e. the 'condition of changeability' is defined as:

$$
y^{\prime}=2\left\lfloor\frac{y^{\prime}}{2}\right\rfloor+b=2\left\lfloor\frac{2 y^{\prime}+b}{2}\right\rfloor+b=2 y^{\prime}+b \leq \min \left(\left(255-x^{\prime}\right), x^{\prime}\right)
$$

Note that both the conditions (condition of expandability (18) and condition of changeability (19)) are same in this case, so, it does not require to check for both the conditions separately, whereas in [2] these two conditions are different and needs separate checking. This in turn reduces mathematical complexity almost halved leading to reduction in implementation cost.

\subsection{Data embedding process}

For a particular fixed threshold value data embedding process are very much similar as in [4] and can be summarized using the following steps. If we want to use this algorithm for region based partitioning method, then first partition the host image into different regions using the steps mentioned in section 4, calculate different threshold value, then apply this algorithm with its corresponding threshold value for a particular region:

1. First the original image is grouped into pair of non- overlapping pixel values. Then forward integer transform (9) is applied to each pair and if it satisfies the condition (17), we will get the average value $x^{\prime}$ and difference value $y^{\prime}$.

2. Now four disjoint set of difference values are formed as described below:

a. EZ: It stands for expandable zero, contains all expandable difference value $y^{\prime}$ having zero value.

b. EN: It stands for expandable non zero, contains all expandable difference value $y^{\prime}$ having non-zeros value, i.e. $y^{\prime} \notin \mathrm{EZ}$.

c. CN: It stands for changeable non zeros value, contains all changeable non zero values of $y^{\prime}$, i.e. $y^{\prime} \notin(\mathrm{EZ} \cup \mathrm{EN})$.

d. NC: It stands for non-changeable values, contains all non-changeable values of $y^{\prime}$.

3. For every difference value belonging to EZ group will be selected for data embedding. Depending on the payload size some selected value belongs to EN group will be selected for data embedding which is placed on subset EN1 and all non- selected value will be placed on subset EN2. For a difference value belongs to subset $E Z \cup E N 1$, bit 1is put into the location map and for subset $\mathrm{EN} 2 \cup \mathrm{CN} \cup \mathrm{NC}$ bit 0 is put into the location map. From location map we can understand any value is expandable (if value $=1$ ) or not (if value $=0$ ) and finally location map has been compressed by using some lossless compression algorithms. 
The International Journal of Multimedia \& Its Applications (IJMA) Vol.4, No.4, August 2012

Original LSB's for difference values in EN2 $\cup \mathrm{CN}$ are then collected and is kept it into an array c as a bit stream.

5. Now location map, original LSB's and payload for those difference values satisfy conditions (17) and (18) are embedded.

6. After embedding all bits, inverse transformation is performed to obtain watermarked image.

\subsection{Data recovery process}

Similarly for a fixed threshold value the decoding and authentication process contains the following steps:

1. The watermarked image is grouped into pair of pixels in a same fashion used during embedding. Forward integer transform is performed to obtain difference and average values.

2. Next two disjoint set of difference value are created as below:

a. $\mathrm{CH}$ : it stands for changeable difference value.

b. NC: it stands for non-changeable difference value.

3. Collect LSB's of all difference value belongs to the subset $\mathrm{CH}$, which form a bit stream $\mathrm{b}$ contains location map, original LSB's and payload.

4. Decode the location map by proper decompressing of the above bit stream and restore the original difference value.

5. Now apply reverse integer transform to get back the original image for authentication.

\section{EXPERIMENTAL RESULTS}

At this section, initially we have presents the performance evaluation of the proposed modified difference expansion [14] algorithm as compared with Tian's algorithms, later on we have considered the three component image watermarking model based on structural information. According to this model image is partitioned into edge, smooth and texture regions. These three regions have experienced different threshold values keeping a fixed mean threshold value to optimize the control distortion. This scheme presents performance evaluation of the proposed algorithm along with relative gain in imperceptivity, payload capacity and security compared to Tian's algorithms [2], [4].

Although we have perform simulation over large number of gray scale images, due to space limitations, we report the same for two test images Lena and Boat as shown in Fig. 1. Fig. 1(a) and Fig. 1 (b) show the original image, Fig. 1 (c) and Fig. 1 (d) show the histogram of the test images. Fig. 2 (a) and (b) shows the watermarked images without region based partitioning method having embedding capacity at $0.0549 \mathrm{bpp}$ and $0.057 \mathrm{bpp}$, the corresponding PSNR values are $44.78 \mathrm{~dB}$ and $44.188 \mathrm{~dB}$, respectively. Fig. 2 (c) and Fig. 2 (d) indicate the watermarked images with embedding capacity at $0.874 \mathrm{bpp}, 0.8698 \mathrm{bpp}$ and their corresponding PSNR values are $39.31 \mathrm{~dB}$ and $37.95 \mathrm{~dB}$, respectively using over embedding.

By adapting over embedding without image partitioning method we have achieved higher embedding capacity up to $1.47 \mathrm{bpp}$ with its respective PSNR and MSSIM values are $33.44 \mathrm{~dB}$ and 0.34 for Lena images, where the payload size is 385320 . Performing similar kind of analysis for Boat images, we have achieved the maximum capacity of $1.24 \mathrm{bpp}$ with its respective PSNR, 
The International Journal of Multimedia \& Its Applications (IJMA) Vol.4, No.4, August 2012

MSSIM and payload size values are $33.4 \mathrm{~dB}, 0.392$ and 325982. Data hiding payload may be increased further, by more over embedding but the visual quality of the images will degrade. As boat image contains more details than Lena image, so it allows less embedding capacity for similar embedding distortion. The highest PSNR values we have achieved for Lena images is $44.78 \mathrm{~dB}$ with its embedding capacity $0.0549 \mathrm{bpp}$ and for Boat image it is $44.18 \mathrm{~dB}$ with embedding capacity $0.057 \mathrm{bpp}$. So, from the above discussion we can conclude, our algorithm is convenient to use both for very high and very low embedding payload.

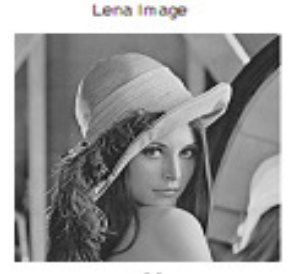

(a)

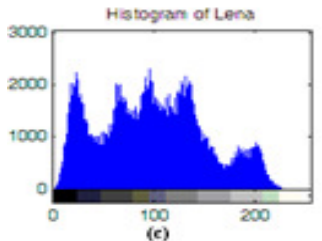

Boat image

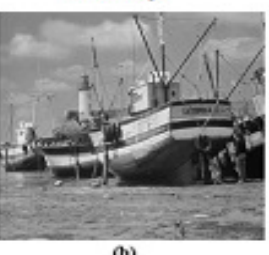

(b)

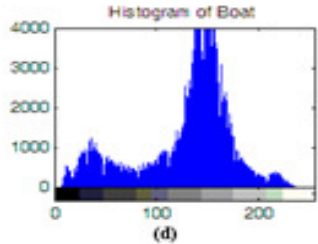

Fig. 1. Test Images (a) Lena (b) Boat; Histogram of (c) Lena and (d) Boat Images.

We also have analyzed performance of the proposed algorithm with and without distortion control as shown in Fig. 3. For comparison we have shown our result with Tian's results under distortion control scenarios. DE with distortion control provides a rhombic shape. For a particular predefined error threshold $(\Delta)$, the larger rhombic area provides larger embedding space. So, from the Fig. 3 it is clear that our algorithm provide larger embedding space than Tian's one.

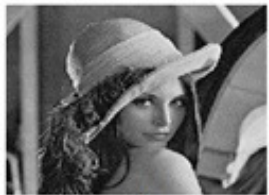

(a)

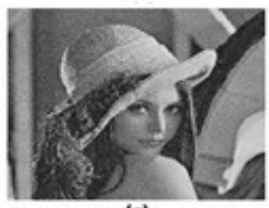

(c)

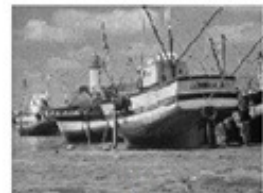

(b)

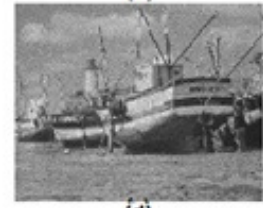

(d)

Fig. 2. Watermarked Images without image partitioning: with Low capacity (a) $0.0549 \mathrm{bpp}$, 44.78dB, payload size (PS) 14404 (b) $0.057 \mathrm{bpp}, 44.188 \mathrm{~dB}$, PS 14970 and with high capacity using over embedding (c) 0.874 bpp, $39.31 \mathrm{~dB}$, PS 229063 (d) 0.8698 bpp, $37.95 \mathrm{~dB}$, PS 228029 for Lena and Boat Image respectively.

The watermarked image produced after transformation should not introduce visual artifacts. By taking the sum and difference of (10), we get $x^{\prime \prime}+y^{\prime \prime}=x+y$ and $x^{\prime \prime}-y^{\prime \prime}=x-y$, which is exactly same with [2]. It indicates that our algorithm preserves both gray level averages and difference between transformed pair of pixels. 
The International Journal of Multimedia \& Its Applications (IJMA) Vol.4, No.4, August 2012
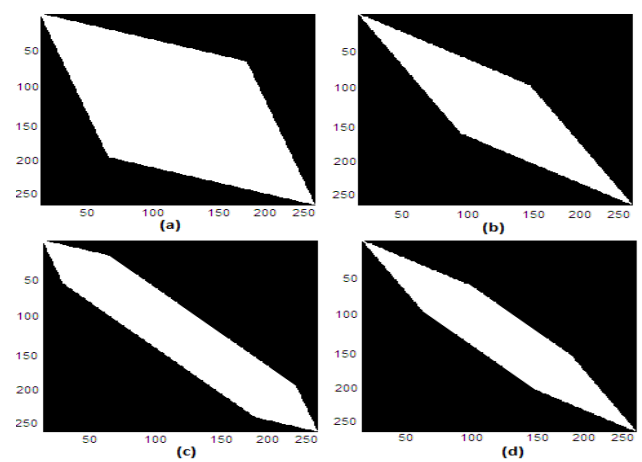

Fig. 3. Distortion control (DC) plot without image partitioning: (a) our algo without DC; (b) Tian's algo without DC; (c) Our algo with DC and (d) Tian's algo with DC.

Furthermore to show the efficiency of our proposed algorithm without considering the image partitioning, the detailed experimental results for Lena and Boat images are given in Fig. 4 and Fig. 5 respectively. In Fig. 4 and Fig. 5 first plot indicates RSNR (dB) vs. watermark payload (bpp), second plot indicates SSIM vs. capacity (bpp) and the last plot is a 3-D plot between SSIM vs. bit rate (bpp) vs. KLD. From Fig. 4 and Fig. 5 it is clear that with embedding rate beyond 0.8 bpp, our results are superior to Tian's for Lena image, whereas it is not that much effective for Boat images as it contains more detail than Lena image as indicated by their histogram shown in Fig.1. The lower value of KLD indicates secured hidden data i.e. statistically invisible. Fig. 4(c) and Fig. 5(c) show the watermarked images (both Lena and Boat image) with different embedding capacity as security for the hidden data is higher compared to [2].
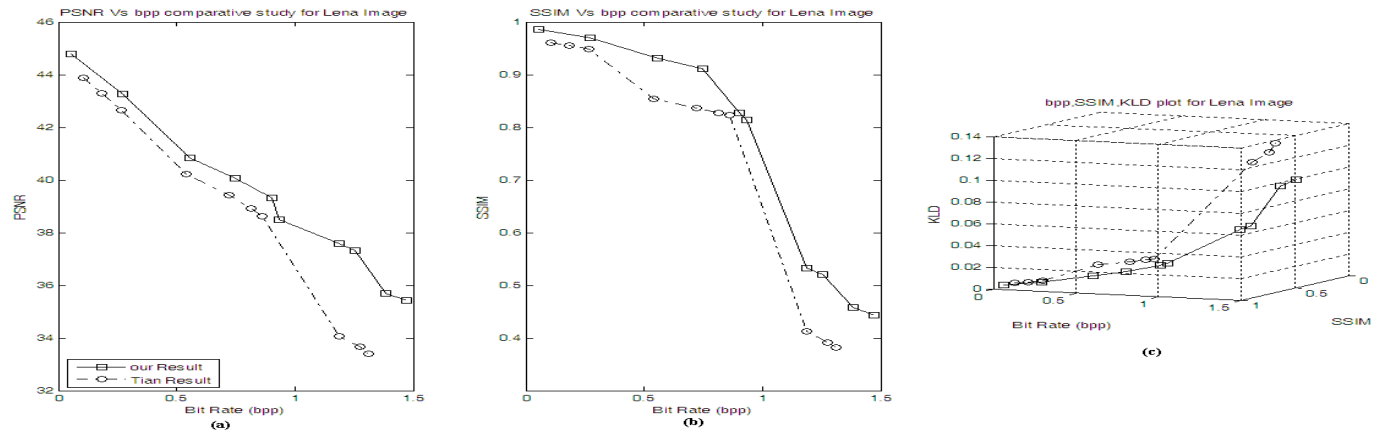

Fig. 4. Result for Lena Images without image partitioning: (a) PSNR Vs bpp; (b) SSIM Vs bpp;

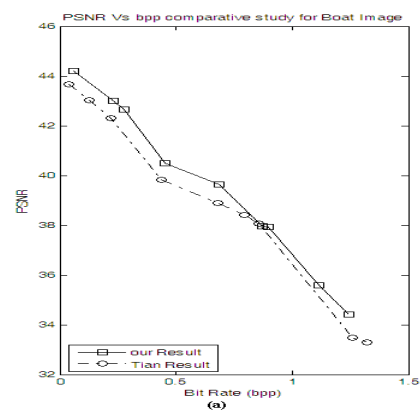

(c) KLD Vs SSIM Vs bpp.
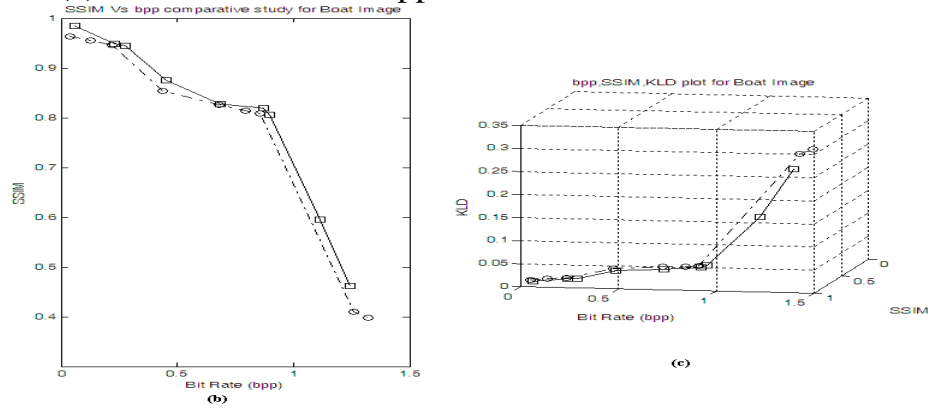

Fig. 5. Result for Boat Images without image partitioning: (a) PSNR Vs bpp; (b) SSIM Vs bpp; (c) KLD Vs SSIM Vs bpp. 
The International Journal of Multimedia \& Its Applications (IJMA) Vol.4, No.4, August 2012

Table 1. Region based threshold and simulation results for edge region.

\begin{tabular}{|c|c|c|c|c|c|c|c|c|}
\hline \multirow{3}{*}{ Image } & $\Delta$ & & & & \multicolumn{4}{|c|}{ Edge Region } \\
\cline { 5 - 9 } & & & $\Delta_{2}$ & $\Delta_{3}$ & $\begin{array}{c}\text { Watermark } \\
\text { length }\end{array}$ & RSNR & SSIM & KLD \\
\hline \multirow{5}{*}{ Lena } & 30 & 26.21 & 46.95 & 16.85 & 38885 & 40.65 & 0.973 & 0.01484 \\
\cline { 2 - 9 } & 35 & 30.58 & 54.77 & 19.66 & 44135 & 40.10 & 0.968 & 0.01563 \\
\cline { 2 - 9 } & 40 & 34.94 & 62.59 & 22.46 & 50454 & 39.47 & 0.962 & 0.01680 \\
\cline { 2 - 9 } & 45 & 39.31 & 70.42 & 25.27 & 57522 & 38.84 & 0.955 & 0.01789 \\
\cline { 2 - 9 } & 50 & 43.68 & 78.24 & 28.08 & 62884 & 38.38 & 0.949 & 0.01861 \\
\cline { 2 - 9 } & 55 & 48.05 & 86.07 & 30.89 & 70470 & 37.79 & 0.940 & 0.01977 \\
\cline { 2 - 9 } & 60 & 52.41 & 93.89 & 33.7 & 75994 & 37.39 & 0.934 & 0.02042 \\
\hline \multirow{5}{*}{ Boat } & 30 & 27.81 & 48.14 & 14.05 & 17701 & 41.51 & 0.991 & 0.01431 \\
\cline { 2 - 9 } & 35 & 32.45 & 56.16 & 16.39 & 20945 & 40.87 & 0.989 & 0.01517 \\
\cline { 2 - 9 } & 40 & 37.08 & 64.19 & 18.73 & 23749 & 40.32 & 0.987 & 0.0158 \\
\cline { 2 - 9 } & 45 & 41.72 & 72.21 & 21.07 & 25535 & 39.95 & 0.985 & 0.01642 \\
\cline { 2 - 9 } & 50 & 46.35 & 80.23 & 23.41 & 28046 & 39.45 & 0.984 & 0.01704 \\
\cline { 2 - 8 } & 55 & 50.99 & 88.26 & 25.76 & 30034 & 39.07 & 0.982 & 0.01682 \\
\cline { 2 - 8 } & 60 & 55.62 & 96.28 & 28.10 & 33371 & 38.46 & 0.979 & 0.01817 \\
\hline
\end{tabular}

Later on we have considered the structural information of an image by partitioning an image into edge, smooth and texture regions by using the algorithm shown in section 4 . Where we have partition an image into three regions using the computed gradient magnitude also we have find out the distortion threshold for individual regions as shown in Table 1 and Table 2.

Table 2. Simulation results for smooth and texture regions.

\begin{tabular}{|c|c|c|c|c|c|c|c|c|c|}
\hline \multirow[b]{2}{*}{ Image } & \multirow[b]{2}{*}{$\Delta$} & \multicolumn{4}{|c|}{ Smooth Region } & \multicolumn{4}{|c|}{ Texture Region } \\
\hline & & $\begin{array}{l}\text { Watermark } \\
\text { length }\end{array}$ & RSNR & SSIM & KLD & $\begin{array}{l}\text { Watermark } \\
\text { length }\end{array}$ & RSNR & SSIM & KLD \\
\hline \multirow{7}{*}{ Lena } & 30 & 38301 & 37.69 & 0.947 & 0.01847 & 3962 & 43.89 & 0.982 & 0.02160 \\
\hline & 35 & 53419 & 36.63 & 0.934 & 0.02127 & 6290 & 43.33 & 0.979 & 0.02221 \\
\hline & 40 & 67694 & 35.83 & 0.921 & 0.02414 & 8224 & 42.82 & 0.976 & 0.02304 \\
\hline & 45 & 85337 & 35.05 & 0.907 & 0.02775 & 10244 & 42.31 & 0.973 & 0.02810 \\
\hline & 50 & 97119 & 34.61 & 0.898 & 0.0332 & 12616 & 41.72 & 0.971 & 0.02810 \\
\hline & 55 & 104709 & 34.34 & 0.893 & 0.03825 & 14620 & 41.27 & 0.967 & 0.02936 \\
\hline & 60 & 111861 & 34.09 & 0.890 & 0.04288 & 19501 & 40.34 & 0.961 & 0.03134 \\
\hline \multirow{7}{*}{ Boat } & 30 & 787 & 39.67 & 0.98 & 0.02297 & 362 & 46.71 & 0.996 & 0.02640 \\
\hline & 35 & 4629 & 38.65 & 0.986 & 0.02161 & 1168 & 46.13 & 0.995 & 0.02751 \\
\hline & 40 & 11602 & 37.28 & 0.968 & 0.02501 & 2097 & 45.52 & 0.993 & 0.02691 \\
\hline & 45 & 35442 & 35.04 & 0.943 & 0.04915 & 3337 & 44.75 & 0.991 & 0.02860 \\
\hline & 50 & 77460 & 33.43 & 0.912 & 0.10033 & 4268 & 44.22 & 0.99 & 0.02956 \\
\hline & 55 & 104825 & 32.83 & 0.896 & 0.13041 & 4950 & 43.84 & 0.989 & 0.02895 \\
\hline & 60 & 115838 & 32.63 & 0.891 & 0.13775 & 6147 & 43.23 & 0.987 & 0.02911 \\
\hline
\end{tabular}

In both the table we have also included the simulation results i.e. SSIM, PSNR and KLD along with the number of watermark bit it can accommodate during data embedding process for individual regions. From both the tables it is clear that by increasing the mean distortion threshold $(\Delta)$ we can embed more amounts of watermark data into each regions as a result the visual distortion get increased so SSIM as well as PSNR values start falling. Also from the analysis of 
The International Journal of Multimedia \& Its Applications (IJMA) Vol.4, No.4, August 2012

the inherent security of the hidden data mentioned by KLD we can conclude that, by introducing more and more watermark bit or extra information within original or host signal the security get disturbed or reduced.

Finally the KLD of the test images using image portioning method with respect to the hiding bit rate without and with control distortion in plotted in Fig. 6. The proposed reversible watermarking technique largely outperforms the previously proposed watermarking scheme. For instance, for Lena image, having mean threshold value 30, 50 and 60, we have got KLD value as $0.018302,0.026638$, and 0.031543 in our scheme i.e. modified DE using image partitioning, whereas in previous scheme(without image partitioning) it was $0.03159,0.03646,0.03782$ respectively.

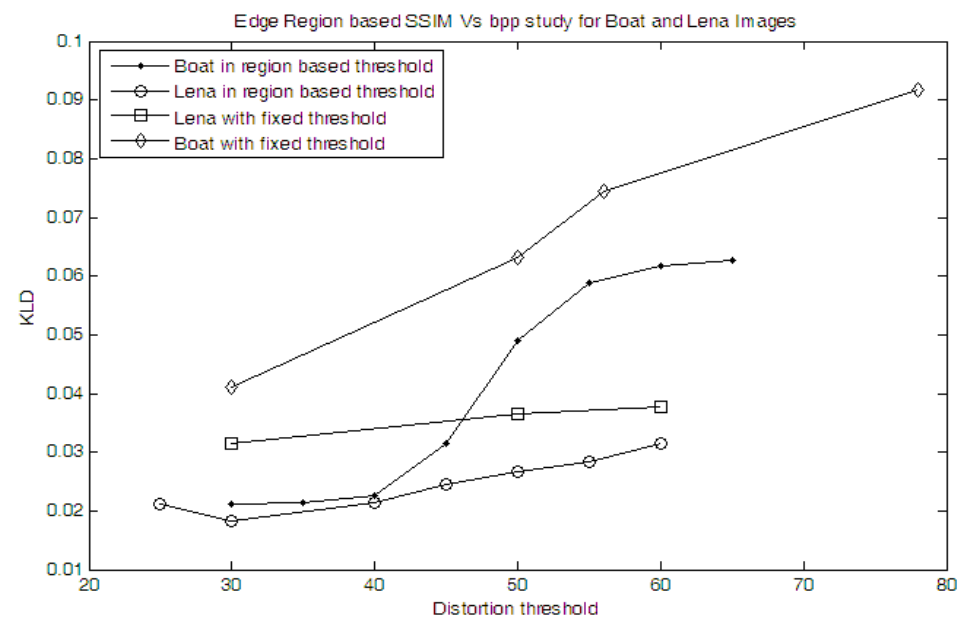

Fig. 6. Security with respect to the distortion control threshold.

\section{CONCluSiONS}

In this paper, a RW scheme has been proposed as an extension of the previously proposed [14] modified difference expansion using gradient based image partitioning method. Using this technique an image is partitioned into edge, smooth and texture regions to control the distortion limited to a mean threshold value over those regions. This structural information based intelligent modified difference expansion scheme exhibits better performance in terms of PSNR and SSIM compare to the previous scheme. Using simulation result it has been shown that the proposed modification not only increases the embedding space and imperceptibility, it also increases the inherent security of the hidden data. Future work may be extended for developing some generalized integer transform to perform reversible operation using DE to optimize the error distortion control in an adaptive manner.

\section{REFERENCES}

[1] R. Caldelli, F Filippine and R Becarelli, (2010) "Reversible Watermarking Techniques: An Overview and a Classification”, EURASIP Journal of Information Security, Vol.2010, Article ID 134546.

[2] J. Tian, (2002) "Reversible Watermarking by Difference Expansion," in Multimedia and Security Workshop at ACM Multimedia '02, December 6, 2002, Juan-Les-Pins, France.

[3] Jen-Bang Feng, Iuon-Chang Lin, Chwei-Shyong Tsai, and Yen-Ping Chu, (2006) "Reversible Watermarking: Current States and Key Issues," in International Journal of Network Security, Vol.2, No.3, PP.161-171, May 2006. 
The International Journal of Multimedia \& Its Applications (IJMA) Vol.4, No.4, August 2012

[4] J. Tian, (2003) "Reversible Data Embedding Using a Difference Expansion," in IEEE Transactions on Circuit and Systems for Video technology, Vol. 13, No. 8, August 2003.

[5] Ran X. and Farvardin N., (1995) "A perceptually-motivated three component image model- part I: description of the model”, IEEE Transaction on Image Processing, Vol. 4, No. 4, pp 401-415.

[6] A. M. Alattar, (2003) "Reversible watermark using difference expansion of triplets," in Proceedings of the IEEE International Conference on Image Processing vol. 1, pp. 501-504, Catalonia, Spain, Sept. 2003.

[7] A. M. Alattar, (2004) "Reversible watermark using difference expansion of quads," in Proceedings of the IEEE International Conference on Acoustics, Speech, and Signal Processing, vol. 3, pp. 377-80, Montreal, Canada, May 2004.

[8] A. M. Alattar, (2004) "Reversible Watermarking Using the Difference Expansion of a Generalized Integer Transform," in IEEE Transaction on Image Processing, Vol 13, No. 8, pp. 1147-1156, August 2004.

[9] X. T. Wang, C. Y. Shao, X. G. Xu and X. M. Niu, (2007) "Reversible Data-Hiding Scheme for 2-D Vector Maps Based on Difference Expansion," in IEEE Transaction on Information forensics and Security, Vol. 2, No. 3, September 2007.

[10] C. C. Lin, S. P. Yang, N.L. Hsueh (2008) "Lossless Data Hiding Based on Difference Expansion without a Location Map,” in 2008 Congress on Image and Signal Processing, 2008, pp. 8-12.

[11] Y. Hu, H. K. Lee and J. Li, (2009) "DE-Based Reversible Data Hiding With Improved Overflow Location Map," in IEEE Transactions on Circuit and Systems for Video Technology, Vol. 19, No. 2, February 2009

[12] Z. Wang, A C Bovik, H R Sheikh, and E. P. Simoncelli, (2004) "Image Quality Asssesment: From Error Visibility to structural similarity," in IEEE Transaction on Image Processing, Vol. 13, No. 4, April 2004.

[13] T. T. Georgiou and A. Lindquist, (2003) "Kullback-Leibler Approximation of Spectral Density Functions," in IEEE Transactions on Information Theory, VOL. 49, NO. 11, Nov. 2003.

[14] H. K. Maity, S. P. Maity and D. Maity, (2012) "Modified Difference Expansion for Reversible Watermarking using Fuzzy logic based distortion control", The Second International Conference on Digital Image Processing and Pattern Recognition (DPPR-2012), 13th to 15th July, 2012, Chennai. 
The International Journal of Multimedia \& Its Applications (IJMA) Vol.4, No.4, August 2012

\section{Authors}

Hirak Kumar Maity received the B. Tech degree in ECE from REC Hamirpur, Himachal Pradesh, India in May 2000 and the M.Tech degree in Microelectronics and VLSI Technology from Jadavpur University, Kolkata, India in 2009. Since 2005, he is associated with the Dept. of Electronics and Communication engineering, College of Engineering and Management, Kolaghat, India. Prior to that he worked as a lecturer in several institutions like, Asansol Engineering College, Asansol, India; Vishveshwarya Institute of Engineering and Technology, Gaziabad, India; Shivdan Singh Institute of Technology and Management, Aligarh, India. His research interest includes Reversible Watermarking, VLSI Deigning and Optoelectronics. Presently he is pursuing his Ph.D from Bengal Engineering and Science University, Shibpur (BESUS), West Bengal, India.

Santi Prasad Maity received his B. E. degree in Electronics and Communication Engineering and M. Tech in Microwaves Engineering, both from the University of Burdwan, in 1993 and 1997, respectively. He received his Ph. D degree in Engineering (Computer Science and Technology) from Bengal Engineering and Science University, Shibpur, India in 2008. During January 2009 to July 2009 and February 2011 to July 2011 he did post-doctoral work concerning watermarking in lured applications in the "Laboratoire des Signaux et Systems (CNRS-Supelec-Universite Paris-Sud 11)" in

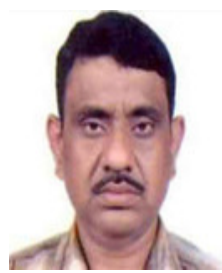
France. He is at present working as Associate Professor and head of the department of Information Technology, Bengal Engineering and Science University, Shibpur since September 2009. Prior that he worked as Assistant professor in the same department from September 2006 to September 2009. He also worked as Lecturer in Electronics and Telecommunication Engineering department of the same university from 2000 to 2006, K. G. Engineering Institute, Bishnupur, bankura, India and Haldia Institute of Technology, haldia, India, from 1997 to 2000. His research areas include Digital Image watermarking, multiuser detection in CDMA, Digital Signal processing, Wavelets for image de-noising, Watermarking, Access Control and Error Concealment, Optimized Spread Spectrum Watermarking and VLSI for Watermarking. He has contributed about 100 research papers in well-known and prestigious archival journals, international referred conferences and chapter in edited volumes 